\title{
A Survey on the Specification of the Physical Environment of Wireless Sensor Networks
}

\author{
Ivano Malavolta \\ Gran Sasso Science Institute, L'Aquila, Italy \\ ivano.malavolta@gssi.infn.it \\ Henry Muccini \\ Information Engineering, Computer Science and Mathematics department, University of L'Aquila, Italy \\ henry.muccini@univaq.it
}

\begin{abstract}
A wireless Sensor Network (WSN) consists of spatially distributed sensor nodes that cooperate in order to accomplish a specific task. What really sets WSNs apart from all the other kinds of distributed systems is the limited processing capabilities of the nodes, contingent energy restrictions, and their strict dependence to physical phenomena like attenuation, reflection, etc. Under this perspective, the physical environment in which WSN nodes are deployed strongly affects the overall quality of the system. Under this perspective, how WSN engineers currently specify the physical environment and how they would like to do it? This paper presents a survey we run by interviewing WSN engineers with a special focus on their practical needs and activities.

By analyzing the collected data, we can conclude that: a) a good number of practitioners describing the physical environment do it by GIS software or informally, b) practitioners not specifying the physical environment do not see a clear return on investment on doing it, c) practitioners rate as (definitely) useful a potential tool for deploying WSN nodes on a virtually specified physical environment.
\end{abstract}

\section{INTRODUCTION}

In a Wireless Sensor Network (WSN), sensor nodes, gateways, and other types of nodes are deployed into a physical area [1]. The exact position where nodes are placed and information about the surrounding obstacles (i.e., everything in the WSN environment that affects the communication between sensor nodes, such as walls, furniture, windows, or small objects in general) and their material could help making an accurate estimate of the physical phenomena that may happen, like refraction, reflection, and attenuation [2]. Such data could allow a more precise measurement of the network performance, in terms of bit error rate, packets loss, and energy consumption, and would enable the prediction of how the WSN will globally behave when nodes are deployed in different ways in the environment.

All this would be possible under the assumption that WSN engineers specify, in some way, the physical environment surrounding the nodes. The main questions we had in mind before starting this study were: do WSN engineers specify the physical environment in some way? In case of a positive answer, how do they specify it and how would they like it to be better specified?

In order to answer those question, we designed a questionnaire structured into six parts and including 28 questions, with the aim of collecting demographical information about the participants, data on how the WSN environment is typically specified, on how the WSN itself is designed, and information on features a tool for WSN environment specification shall include. The questionnaire has been completed by 21 engineers from 18 different organizations in 9 countries, that have been involved in WSN development. We got a number of interesting results that are presented in Section III.

The paper is structured as follows: in Section II we describe the research questions, identify the survey target population, and describe how the questionnaire has been designed. Section III gives an overview of the results we got, while reflections are presented in Section IV. Threats to validity are discussed in Section V, and Section VII concludes the paper and briefly discusses future work.

\section{Design of the Study}

In this section we describe the main research questions we want to investigate in our study (Section II-A), then we characterize the process we followed for selecting a representative sample of WSN engineers (Section II-B), and finally we describe how we designed our survey instrument (Section II-C).

\section{A. Research Objectives}

Our goal is to better understand the current practices followed by WSN engineers for modeling the physical environment where WSN nodes are deployed. Then, we want to analyze the perceived strengths, limitations and needs associated to the state of the art on WSN physical environment specification. The results of this study will (hopefully) lead to the development of new approaches and best practices for specifying the physical environment of a WSN.

We designed our research with this question in mind: How WSN engineers currently define the physical environment, and how they would like to do it? Indeed, having a clear definition of the physical environment of a WSN can be specially useful for developers and system engineers when they have to reason about (i) the network topology, (ii) the presence of possible physical obstacles (e.g., walls, trees) within the network deployment area, (iii) how much power is consumed by the application running on the nodes with respect to the 
used batteries or harvested energy sources, (iv) how well an area is monitored or tracked by sensors, and so on.

The above mentioned research question can be elaborated into the following research sub-questions:

- RQ1: Do engineers explicitly specify the physical environment where the WSN is going to be deployed? If so, how do they accomplish this task (e.g., formally, informally, etc.)?

- RQ2: Do engineers specify the sensor nodes and their exact position within the physical environment of a WSN? If so, how do they do it (do they consider obstacles, hardware configuration, etc.)?

- RQ3: What are the most relevant features a potential tool for specifying the physical environment of a WSN shall expose? Does the tool have to consider the exact shape of the obstacles, or an approximation is satisfactory? How would WSN engineers prefer to interact with such a potential tool?

To answer these research questions, we designed an on-line questionnaire. In designing the study, we paid particular attention to the selection of participants which are a representative sample of WSN engineers, both in academia and in industry. In the following section we will describe how we selected a representative sample.

\section{B. Identification of the Target Population}

We began by identifying the target population of our survey. The profile of the participants of our study is the following: "Engineer who has been concretely involved in the development of at least a wireless sensor network in the last ten years".

Once we profiled our target population, we applied a combination of convenience and snowball sampling for selecting the participants to our study [3]. Firstly, we directly selected WSN engineers from our personal contacts and we looked for other contacts available on reference websites, newsgroups, and other web resources about WSN operating systems, node vendors, and WSN technologies in general. Secondly, we asked selected participants to nominate additional experts in their network (following a snowball sampling approach [3]). Fundamentally, we have been forced to use this non-probabilistic sampling method for two reasons: i) the target population is actually very specific and of limited availability since WSN engineering is still not a well settled discipline, and ii) WSN engineers are also typically involved in other activities like research, development of other kinds of systems, etc.

As a result, we sent 54 emails in total and eventually 21 WSN engineers from 18 different organizations in 9 countries participated to the survey. The main affiliation types of respondents range from university (14), to center of excellence (2), company (2), research institution (3). This type of population is not surprising since WSN engineering is still not a well settled discipline and many researchers on WSNs are also practitioners in the field and involved in professional activities.

\section{Design of the Questionnaire}

As already anticipated, we designed the questionnaire in order to find a question to our $R Q 1, R Q 2$, and $R Q 3$ research questions. The questionnaire is composed of 28 questions: 21 are closed-ended questions, whereas the rest are open-ended questions $^{1}$. The rationale behind this choice is twofold: (i) open-ended questions may be subject to misinterpretations containing potentially irrelevant or confusing answers, and (ii) closed-ended questions are easier to code and analyze. We decided to leave open-ended questions only in those cases when it was strictly needed, allowing respondents to freely discuss their individual experiences.

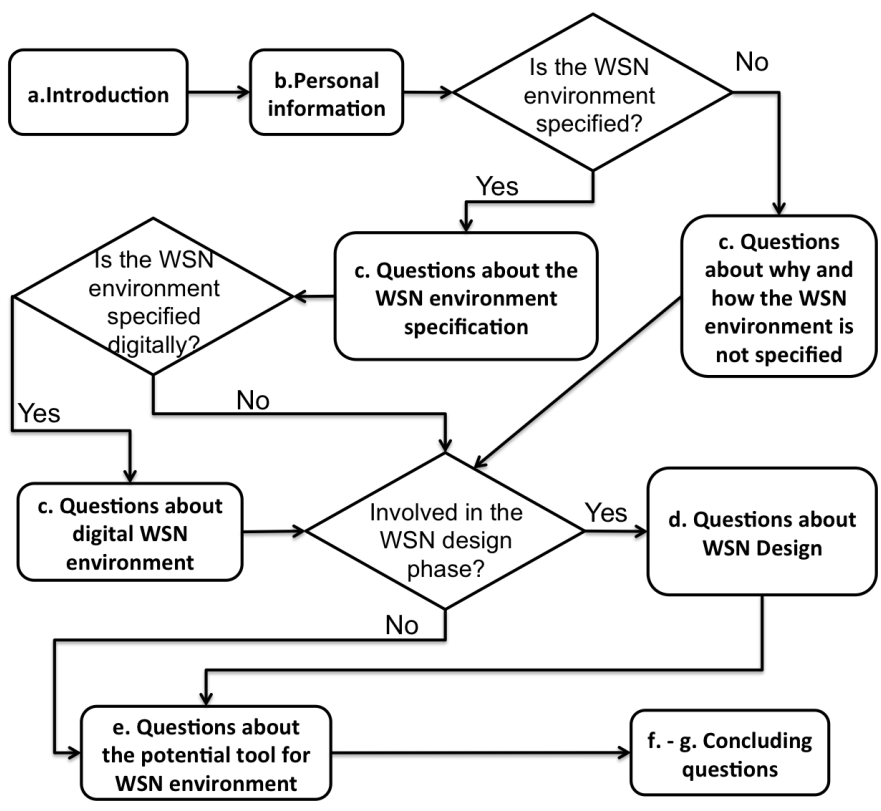

Fig. 1. Questionnaire flow

The questionnaire is structured in six sections (a-f) and follows the flow shown in Figure 1. Boxes are labelled with the research questions they address. The six sections are:

a) Introduction. This section provides general information about the study and terminology that we use throughout the questionnaire (such as, WSN, WSN environment, deploy, obstacle, etc.). We also explain the purpose of our study (see Section II-A) and other administrative information.

b) Personal information. These questions allow us to understand the working environment and professional background of the participant. The data collected in this section is used to gather demographical information about the participants.

c) WSN environment specification. In this section the focus is on how the physical environment is specified during the various development activities of a WSN. Firstly, we ask participants whether they explicitly specify the environment in their design and development

\footnotetext{
${ }^{1} \mathrm{~A}$ transcript of the questionnare is available here: http://www.di.univaq.it/ malavolta/wsn/WSNenv.pdf
} 
workflow. If the answer is negative, then we ask why it is not considered and how they proceed to the deployment of a WSN without defining the physical environment of the WSN. If the answer is positive, then we ask further details.

d) WSN design. In this section we focus on the specification of the sensor nodes and their positioning within the physical environment of the WSN. Thus, firstly we ask whether the participant is directly involved in WSN design activities. If so, we ask additional questions about its activities. We also ask participants if and how they consider physical obstacles that may exist in the deployment area of the WSN (e.g., walls, floors, windows, large- and small- sized objects, etc.) during the design of the WSN. Then, we ask participants to rate the usefulness of having a hardware specification of the WSN nodes (by hardware specification here we mean information about battery voltage, radio communication devices, etc.). Finally, we ask how they evaluate if the sensor nodes positioning in the environment is adequate.

e) Potential tool for WSN environment. In this section we ask participants to provide guidelines on how to build a potential tool for virtually deploying a WSN in the physical environment; such a potential tool could simulate an environment where to virtually deploy a set of defined sensor nodes. More specifically, we ask them to rate the usefulness of such a potential tool. Then, we ask how they would prefer to interact with such a potential tool.Moreover, we ask participants whether they are interested in defining the exact shape of the obstacles or to approximate the shape of the obstacles (e.g., a table may be rendered as a cube). Finally, we ask participants to rate the importance of considering the following physical effects when defining a WSN: attenuation, refraction, diffraction, reflection, polarization, scattering.

f) Conclusion. In this section we seek any additional information that participants would like to add. According to our snowball sampling method, we also ask the participant to list the names of colleagues who can be interested to participate in our study.

\section{RESULTS}

This section reports on the main results we extracted from the collected data. Following the questionnaire structure described in Section II-C, this section describes the outcomes related to the four main sections, from $\mathrm{b}$ to $\mathrm{e}$.

\section{A. Population}

The participants of this survey are 21 engineers from 18 different organizations in 9 countries. Among those participants, 14 have an experience of more than five years in WSN development, whereas the experience of the remaining participants is under five years. If we consider that the WSN application domain is relatively young, our population sample is good enough to be confident about the answers provided in the remaining of the questionnaire.

When asked about how many projects involving WSN development they have worked on, the majority of participants (about 53\%) declare to have worked on less then three projects. Other participants declare that they have worked on more than six (about 23\%) and between three and six (about 19\%) WSN projects in their career; the remaining $5 \%$ of participants do not give any relevant information on this aspect of their career. This is still a good result, since we can be reasonably confident that the provided answers come from people actively working in the field.

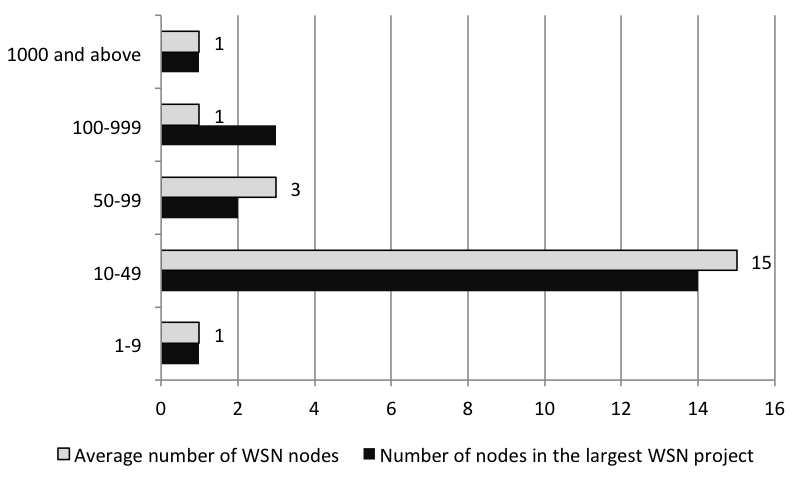

Fig. 2. Number of nodes in past WSN projects

In the remaining of this section of the questionnaire, we ask participants three questions about the kind of WSN applications they have been working on. The first two questions are about (i) the number of nodes that have been included in average in the WSNs they have worked on, and (ii) the number of nodes that have been included in the largest WSN (in terms of number of nodes) they have worked on. Figure 2 shows a graph summarizing the answers provided by participants.

Fundamentally, the main message of the chart in Figure 2 is that the majority of the participants has worked in smallmedium WSN projects (i.e., those involving networks with ten to forty-nine nodes). Even if their number is very much smaller, the other kinds of network sizes are homogeneously distributed across the remaining participants.

The final question of this section is about the kind of wireless sensor network the participant has worked on in his career. More specifically, in this question we are focussing on the distinction between indoor and outdoor WSNs. When looking at the answers of this specific question, we find out that there is a certain balance among the answers. Indeed, about $43 \%$ of participants declare that they equally worked on indoor and outdoor WSNs, and about 24\% and 28\% declare that they mostly worked on indoor and outdoor WSNs, respectively. Nonetheless, only one respondent out of twentyone declare to work on indoor WSNs only, and nobody declares to work on outdoor WSNs only. 


\section{B. WSN environment specification}

When asking if the WSN environment is explicitly specified during the design and development of the WSN application, responses of the participants are almost equally distributed (see Figure 3). Ten respondents out of twentyone answered positively, and the other eleven respondents answered negatively. If on one side this result is encouraging for our study since we can investigate on both type of development processes, on the other side we can only report this data, without drawing any strong conclusion on this aspect.

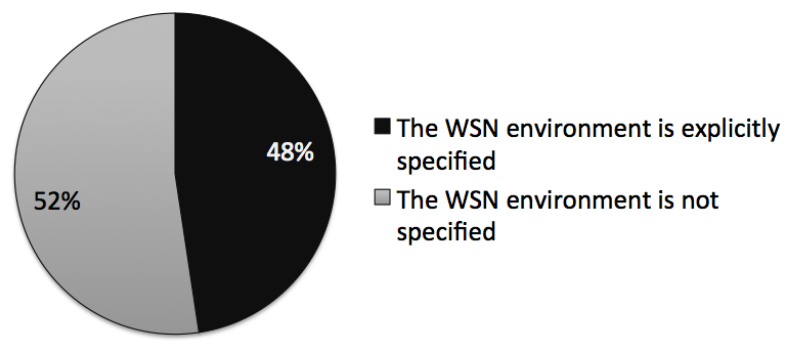

Fig. 3. Explicit specification of the WSN environment

The main reasons brought by respondents for not explicitly considering the WSN environment are: (i) no perceived usefulness in having an explicit representation of the WSN environment (about 54\%), and (ii) the lack of satisfactory tools, algorithms or models for specifying the WSN environment at the right level of detail (about 46\%).

Moreover, when asked about how they proceed to the deployment of a WSN without defining an environment, participants indicated that they typically work on WSN that can adapt to the environment they are deployed in, so that they do not need to know a priori their environment (about $37 \%$ ), only perform measurements of the WSN on the field after deployment (about $27 \%$ ), only perform preliminary measurements of the area and network simulation before actually deploying the WSN (about 27\%), make estimation based on their professional experience (about 9\%). These results show that researchers (i) could work on this issue providing a more concrete evidence about the usefulness of explicitly representing the WSN environment (e.g., by providing experimental results), (ii) have to further work on methods, algorithms, and tools for better supporting the definition of the environment of the WSN. The authors of this paper are currently investigating and working on this issue.

Among those participants explicitly considering the WSN environment, $60 \%$ of the respondents declare that they are in charge of specifying the environment (either alone or within a group), and $40 \%$ of the respondents declare that someone else specifies the environment. Among those participants explicitly considering the WSN environment (see Figure 5), we see that a good portion of respondents (summing up to $40 \%$ ) precisely specify the WSN environment, others equally specify the environment either precisely or as a draft (about $30 \%$ ), a smaller portion of respondents always define the environment as a draft (about 20\%), the remaining $10 \%$ of

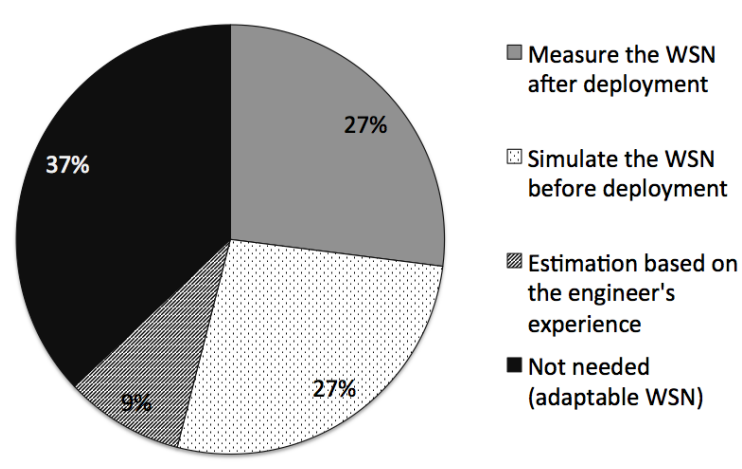

Fig. 4. How some engineers deploy a WSN without defining the environment

respondents do not provide any information about this aspect ${ }^{2}$. By looking at the number, we see a major trend in specifying the environment in a precise way, rather than relying on draft specifications.

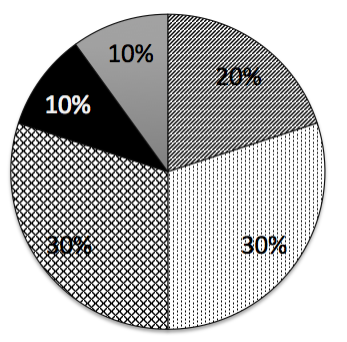

\author{
Always by a draft \\ Mostly by a precise specification \\ Equally \\ - Not specififed \\ $\square$ Always by a precise specification
}

Fig. 5. Specification of the WSN environment in the last project

When dealing with the distinction between digital and paper-based representations, there is a clear trend in favour of the digital representation (about 90\%). By investigating further on the digital representation (see Figure 6), respondents mostly rely on maps and geographic information systems (about 40\%), followed by those who use office software, such as Powerpoint, Omnigraffle, Word (about 30\%), finally other respondents declare to always use dedicated software, such as some ad-hoc software provided by the manifacturer of the nodes (about 20\%), the remaining $10 \%$ declare not to have a direct knowledge about the used software.

For what concerns the used file formats, they fall into two main categories: text-based representations (about 40\%) and images $(30 \%)$. Basically, those results uncover the great variance when considering which software is used to represent the WSN environment.

When considering the 2D-vs-3D issue, the majority of respondents (about 80\%) declare to specify the environment in $2 \mathrm{D}$, followed by the $10 \%$ of respondents declaring to use a $3 \mathrm{D}$ one, and those declaring to use a combination of the two (about 10\%). 2D representation is the clear winner here. This may be due to the complexity of producing 3D models, or to the fact that $2 \mathrm{D}$ models are perceived to be sufficient for

\footnotetext{
${ }^{2}$ It is important to note that in this question we explicitly ask respondents to focus on their last project.
} 


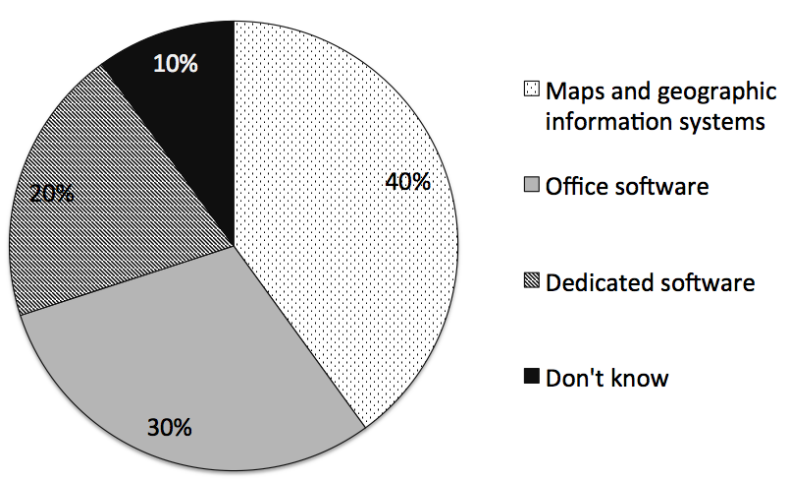

Fig. 6. Used digital representation of the environment

representing the environment of a WSN; unfortunately, we do not have enough information to sharply distinguish between these two reasons. However, when asked about what is the best option among the $2 \mathrm{D}$ and the 3D ones, about $50 \%$ of respondents declare that the best option would be to use a combination of $2 \mathrm{D}$ and $3 \mathrm{D}$ representation, followed by the $30 \%$ of respondents exclusively preferring the $3 \mathrm{D}$ one. In this case, $3 \mathrm{D}$ representation is the main trend.

\section{WSN design}

In our questionnaire, WSN design has been defined as the definition of the sensor nodes and how they are positioned in the physical environment. The majority of the respondents declare to be directly involved in the WSN design phase (about $71 \%$ of participants). This is an important result since it validates all the answers provided in this section.
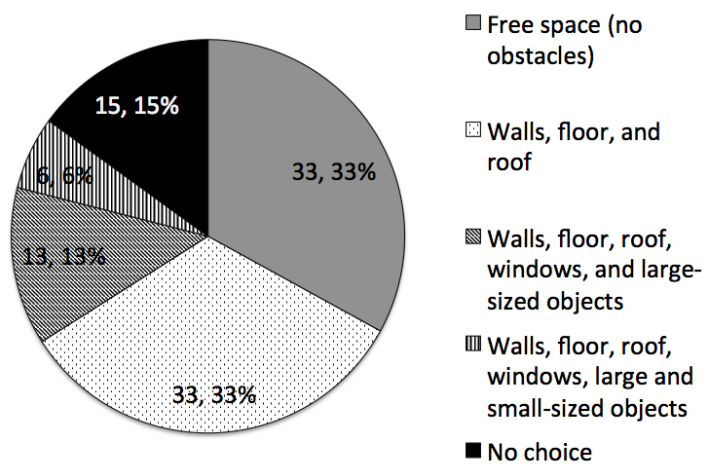

Fig. 7. Types of considered physical obstacles

The first question of this section is about the types of physical obstacles considered during the WSN design phase (see Figure 7). About $33 \%$ of assume not to have any obstacle in the environment while designing the WSN; about another 33\% of respondents declare to consider the presence of walls, floor and roof while designing the WSN. Moreover, about $13 \%$ of respondents declare to consider also the presence of windows and large-sized objects (e.g., furniture, sofa, wardrobes), and other $6 \%$ of respondents declare to consider both large-sized objects and small-sized objects (e.g., laptop, TVs, lamps). The remaining $15 \%$ of respondents were unable to pick a decision among the provided answers.

WSN engineers must address challenging extra-functional requirements such as performance, security, energy consumption; in order to achieve the demanded high level of efficiency, the software of a WSN application is closely tied to specific hardware platform of its nodes. When asked about the usefulness of having a hardware specification of the sensor nodes of the WSN, about $94 \%$ of respondents declare that it is definitely useful. We were expecting this, since WSN engineers must have at least some knowledge about the hardware features of the nodes used in the WSN. For example, information about the transmission power of the antenna, available sensing and actuating devices, batteries voltage, are necessary to effectively design and develop a wireless sensor network.

In most cases, each node of a WSN communicates via a radio channel and it is powered by a battery. The most important resource of a WSN node is energy, and it determines the lifetime of a WSN. The positioning of the nodes of a WSN is crucial for its life time, and it depends on factors like the distance between the nodes, the presence of physical obstacles, the used routing protocol (single-hop, multi-hop, with clustering, etc.), the presence of physical effects such as refraction, diffraction, reflection, absorption, etc. Because of the presence of such a high number of interrelated factors, estimating the best positioning of the nodes of a WSN that maximizes network life time is a hard problem. The concluding question of this section is about the instrument engineers use for evaluating if the sensor nodes positioning in the environment is optimal/adequate for the system. In this case, the majority of respondents (about 87\%) declare that they evaluate it by experimentation. That is, they place some test node in the physical environment, they measure their performance in terms of some parameters (such as, bit error rate, packets loss, etc., energy consumption, etc.), and then they try to predict how the WSN will globally behave with respect to those parameters. This result is quite interesting. We will discuss it in Section IV.

\section{Potential tool for WSN environment}

In this section we ask participants to reason, based on their experience, on a potential tool that allows engineers to virtually deploy a WSN in the environment. Such a potential tool could simulate an environment where to virtually deploy a set of defined sensor nodes into a digital version of its physical environment.

Firstly, we ask participants to rate the usefulness of such a potential tool. Figure 8 graphically shows the distribution of the provided answers. Here the results are clear, the majority of participants perceive the tool as useful (summing up to about $85 \%$ of participants), and only three respondents basically declare to do not see its usefulness.

Moreover, we go further with the questionnaire by asking participants, assuming that such a tool exist, how they would prefer to interact with such a potential tool (see Figure 9). 


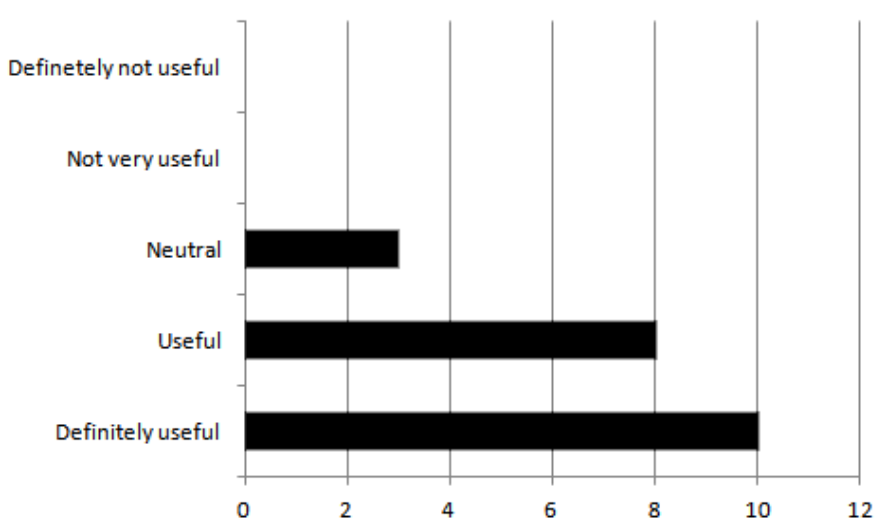

Fig. 8. Usefulness of a tool for virtual deployment

In this case provided answers are almost equally distributed. About $23 \%$ of participants answer that they would prefer to create a file with a dedicated tool (we assume they referring to either CAD or GIS software in this case) and then to import it into the virtual deployment tool; instead about $33 \%$ of participants answer that they would prefer to directly specify the environment within the virtual deployment tool (e.g., by means of a suitable drawing interface). Finally, about $42 \%$ of participants declare that they would prefer a combination of the two previous options, that is by firstly importing an image file to be used as a guide to the drawing phase within the virtual deployment tool.

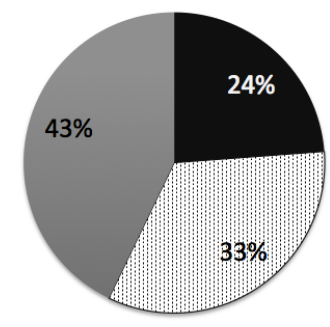

\footnotetext{
By importing a file produced by means of an external tool (for example Autocad)

By directly drawing the environment within the too

By firstly importing an image file to be used as a guide to the drawing phase within the tool
}

Fig. 9. WSN environment tool interaction

For what concerns the definition of obstacles in the environment, we do not see any strong trend towards some commonly accepted preference. Indeed, when asked about whether they would be interested in defining the exact shape of the obstacles when defining the WSN environment, the majority of participants (about 48\%) do not have a clear opinion on this aspect; moreover, the remaining answers are fundamentally equally distributed: $24 \%$ declare that this feature is interesting, whereas $28 \%$ declare it is not.
Finally, we ask participants to rate which physical effects are most important to consider when defining the physical environment of a WSN. For each physical effect, participants slightly differ in their average opinions about its importance when considering the environment of a WSN. Table I shows the results provided by participants. The table shows the participants' answers, in Likert scale, from -2 (least important) to 2 (most important). In order to better understand the obtained values, we ranked them based on a weighted sum over each row of the table (see the ws column).

\begin{tabular}{lcccccc} 
Physical effect & $\mathbf{- 2}$ & $\mathbf{- 1}$ & $\mathbf{0}$ & $\mathbf{+ 1}$ & $\mathbf{+ 2}$ & ws \\
\hline Attenuation & 0 & 0 & 0 & 7 & 14 & $\mathbf{7}$ \\
Reflection & 0 & 0 & 2 & 11 & 8 & $\mathbf{5 . 4}$ \\
Scattering & 0 & 1 & 6 & 7 & 7 & $\mathbf{4}$ \\
Diffraction & 0 & 2 & 6 & 11 & 2 & $\mathbf{2 . 6}$ \\
Refraction & 0 & 4 & 5 & 9 & 3 & $\mathbf{2 . 2}$ \\
Polarization & 0 & 4 & 8 & 7 & 2 & $\mathbf{1 . 4}$
\end{tabular}

TABLE I

IMPORTANCE OF PHYSICAL EFFECTS FOR THE WSN ENVIRONMENT

Basically, all the physical effects are generally perceived as important, with some more visible preference for attenuation (summing up to $100 \%$ rated as important or definitely important) and reflection (about $90 \%$ rated as important or definitely important).

\section{REFLECTION}

The survey presented in this paper has been centred around three main research questions. The findings are summarized in the following.

RQ1 asks "Do engineers explicitly specify the physical environment of a WSN? If so, how do they do it (e.g., formally, informally, etc.)?". The results of our study reveal that, so far, WSN engineers are quite divided with respect to whether they create an explicit definition of the physical environment. Indeed, if on one side there is a good number of engineers describing the physical environment, there is still an (almost equal) number of engineers who do not, mainly because they do not see a clear return on investment on doing it, or because they do not find any satisfactory tool or method to do it. This result shows that researchers (i) could work on this issue providing a more concrete evidence about the usefulness of explicitly representing the WSN environment (e.g., by providing experimental results), (ii) have to further work on methods, algorithms, and tools for better supporting the definition of the environment of the WSN. The authors of this paper are currently investigating and working on this issue [4]. Also, when considering the formal-vs-informal issue, we notice that the majority of participants prefer to (i) define the physical environment via mapping and geographic information systems, and (ii) to provide the description of the environment as a combination of text and images. This is a good indication 
for future research and development direction towards a mapbased instrument for defining the physical environment of a WSN; also, participants declare that they would prefer to see a combination of 2D and 3D representation of the environment.

RQ2 asks "Do engineers specify the sensor nodes and their exact position within the physical environment of a WSN?". The results of our study uncover that WSN engineers typically either (i) assume to do not have any obstacle in the environment while designing the WSN (i.e., free-space environment), or (ii) consider only very large obstacles, such as walls, the floor and the roof. Also, when asked about how they evaluate if the sensor nodes positioning is adequate for the system, the majority of respondents declare to rely on physical testbeds where sensor nodes are placed in a controlled real-world environment and measured. All the above mentioned results may indicate that current simulation and analysis techniques demand too much effort to WSN engineers, both in terms of learning time, specification complexity. In light of this, we report also two quotations of the respondents that summarizes this explanation:

"Simulation is performed only if simple, feasible and meaningful, otherwise deployment".

"Usually the available simulation tools do not provide a functionality to define and describe the environment. However, I feel that it is equally important to describe the environment and its behaviour in addition to the models that define how the networking part will function. I believe this is due to the difficulties in defining accurate models for the environment."

RQ3 asks "What are the features of a potential tool that allows engineers to specify the physical environment of a WSN?". The results of our study reveal that a WSN engineers strongly need a tool for (i) specifying the physical environment of a WSN, and (ii) virtually deploying WSN nodes into it. That tool could allow engineers to specify the environment in different ways, e.g.: by importing a map developed with a CAD tool, by drawing it directly within the tool itself, by importing an image that will serve as the basis for a subsequent drawing phase. Under this perspective, we do believe that the latter option provides a good trade-off in terms of level of usability, simplicity and preciseness in the definition of the obstacles. For what concerns the physical effects that should be considered in such a potential tool, attenuation is the clear winner, followed by reflection. So, we can conclude that they constitute the minimal set of physical phenomena that should be considered when using the potential tool for defining a physical environment for WSN.

\section{THREATS TO VALIDITY}

We took great care in all the phases of the study in order to avoid biases as much as possible. More specifically, we have been very careful in selecting the sampling method, in designing the flow of the questionnaire, in wording the questions so that they are understandable to the target audience, and especially in analysing and reporting the obtained results.

Also, we carried on two pilot tests in order to simulate the survey instrument in a controlled environment [5]. We revised the questionnaire to reflect the received inputs.

In the following we discuss the threats to validity that our study may have suffered, and how we tried to mitigate them.

\section{A. Internal Validity}

The only instrument we used for conducting our study is the on-line questionnaire. That is, we firstly contacted potential participants by email asking them if they were able to participate to our study, then we sent them the link to the on-line questionnaire; this allowed participants to fill in the questionnaire as soon as they have time to do it, and to reason about their answers as long as they wished. However, we could not directly interact with participants, i.e., we could not ask follow-up questions to the participants who fill the questionnaire. We tried to mitigate this possible bias by (i) providing a set of definitions at the beginning of the questionnaire that are used consistently in the survey to help them to answer the questions, and (ii) asking an open-ended question at the end of questionnaire in which participants may freely give their comments on the questionnaire and on WSN engineering activities in general.

\section{B. External Validity}

We administered our survey to 21 participants across the world, we acknowledge that the sample size of our study is quite limited and that this prevents us to perform a deep statistical analysis of the obtained data [6]. As already described in Section II-B, this limitation is mainly due to the specificity ot the target population and to its limited availability (mainly because WSN engineering is still not a well settled discipline). In light of this, we are planning to work on a future research involving larger data samples that will be able to assess its statistical significance and validity. We are planning to work on larger data samples by employing other means of communication for contacting potential participants (e.g., via phone calls, mailing lists, etc.), by collecting direct contacts via a dedicated mini-website presenting the results of this work (in this case, visitors could be asked to sign up to our mailing list), by actively intercepting participants to conferences and events related to the WSN field, etc. The results of the survey with a larger sample size will be interesting to look at from the perspective of deploying wireless sensor networks. Finally, we will assess whether the statistical analyses of our future survey will change our findings as well.

Moreover, a possible threat to validity regards the instrument we chose for our study. Indeed, performing in-depth interviews could have resulted in a deeper investigation on the topic. We decided to perform a questionnaire-based survey mainly because of (i) budget constraints related to both time and available resources, and (ii) the availability of participants: some participants overseas are not contactable during the normal working hours and we had to interact with them only via email. We are aware that in our survey we could not ask follow-up questions to the participants who filled in the questionnaire to clarify unclear answers. In order to mitigate this possible bias, we took care of the following aspects: 
- when reasonable, we asked participants to provide a detailed explanation of the given answers by means of follow up open-ended questions;

- we applied a coding procedure on the detailed explanation of the given answers, in order to gain deeper insights into the issue being investigated.

As described in Section II-B, in this survey we applied a combination of convenience and snowball sampling. If on one side these two methods help us in selecting participants in a very precise and accurate manner, on the other side they may risk to be biased (i.e., not being representative of the target population) [3]. However, participants come from different professional background, they are from 9 countries and 18 different organizations. This indicates a good spread of participants' profiles to increase the confidence that our results do not suffer from relevant biases with respect to the sampling method we use. Also, this mitigates the possible bias of having a small sample, counting only 21 participants.

\section{RELATED WORK}

This section, while not having the pretension to be complete and to cover the full multi-disciplinary components of this topic, reports on some relevant research work related to the physical environment modeling of a WSN. It has been elaborated by taking into account the most relevant papers and significant inputs provided by colleagues actively working in the WSN and modelling topics.

In [7], Maissa et al. present a domain specific modeling language (DSML) for the design and analysis of WSN. The DSML defines four different models. Among them, one is the physical environment model that defines physical quantities as a function of space and time. The deployment model specifies how instances of nodes are spread into the physical environment.

In [8] Samper et al. propose the GLONEMO formal model for the analysis of ad-hoc sensor networks. In this work, a constraint-based language named Lucky is used to model the environment. Samper et al. in [9], instead, analyze the influence of an environment (and its model) into the analysis of a WSN. However, their focus is not on the physical environment, but on a more general conception of the environment, including the set of stimuli that may appear around a WSN.

An explicit definition of the physical environment is presented in [4], where the proposed tool allows engineers to model through an ENVML model, real-world dimensions in a $2 \mathrm{D}$ space, and obstacles with attenuation coefficients that may represent a specific material.

In [10] the authors make use of an informal, graphical, description of a 3D physical environment, where multiple type of sensors are deployed. While the paper does not focus on the physical environment modeling, still, it shows the researchers need to model a 3D view of the environment.

In the survey in [12] the authors have proposed an enhanced propagation model which takes into account single- and multiwall and floor attenuation factors for indoor environments. The survey also refers to [13], where the environment is divided into triangular regions. Still, there is no reference to modeling languages specific for the physical environment.

By comparing the practitioners' needs reported in Section III with the solutions discussed in this section, we can come up with the reasoning that academic research does not seem to be in line with practitioner needs. Practitioners, in fact, report that they do not explicitly model the physical environment of a WSN (52\% of the respondents), since they still miss satisfactory tools, algorithms or models for specifying the WSN environment at the right level of detail. A discrepancy between the state-of-the-art and practitioner needs can be also elicited in other places: practitioners use maps, geographic information systems, powerpoint and other means to specify the environment, and would like to represent it through CAD or GIS software, or by using a suitable drawing interface. Current scientific work are more keen to provide mathematical models to describe the environment, instead. Where the distance between state-of-the-art research and practitioners' needs is closer is on the evaluation of the sensor nodes positioning in the environment. The majority of respondents (about 87\%) declare that they evaluate it by experimentation, by placing some test node in the physical environment and then trying to predict how the WSN will globally behave with respect to those parameters. Our understanding is that there exists stateof-the-art methods and tools that could support practitioners in this task. However, a stronger link between researchers and practitioners shall be built.

\section{COnClusions And Future Work}

This paper presents the first investigation on how the physical environment of WSNs is currently specified in practice. We hope our colleagues will find these results useful for building new solutions and methods for specifying the physical environment of a WSN.

During the analysis of the collected data we noted that (i) a potential instrument that allows WSN engineers to specify the physical environment of a WSN is actually needed (see Figure 8). Also, it emerged that practitioners could prefer to specify the environment by firstly importing an image representing the environment visually, and then to use it as the basis for a subsequent drawing phase (see Figure 9). We are currently in the process of building such an instrument as a dedicated component of the WSN modeling framework presented in [4].

Moreover, our modelling environment proposes a dedicated domain-specific modeling language (DSML) in which the environment is represented as an area in the $2 \mathrm{D}$ space; this choice is motivated by the observation that currently the majority of respondents declare to specify the environment in $2 \mathrm{D}$, so we decided to follow this trend in order to present a modelling environment which is, at the same time, familiar to practitioners and simple to be used.

Also, in our DSML any kind of relevant obstacle can be placed in the environment. Each obstacle is characterized by its material (e.g., concrete wall, wooden door, glass, etc.) and its attenuation coefficient; this solution is rooted in the clear 
interest of practitioners to the attenuation physical effect when defining the physical environment of a WSN (see Table I). The shape of an obstacle is given by a sequence of coordinates representing its perimeter in the 2D space. In our DSML, areas identify portions of environment in which nodes of the same type can be distributed accordingly to a distribution policy, this provides a good solution for virtually deploy a large number of nodes in a straightforward manner.

\section{ACKNOWLEDGMENTS}

We would like to thank the engineers and developers who participated to this survey. This work is partly supported by the Ricostruire project (RIDITT - Rete Italiana per la Diffusione dell'Innovazione e il Trasferimento Tecnologico alle imprese).The authors would like to thank Lorenzo Salvatore, for his help and support in making this work possible.

\section{REFERENCES}

[1] L. Mottola and G. P. Picco, "Programming wireless sensor networks: Fundamental concepts and state of the art," ACM Comput. Surv., vol. 43, pp. 19:1-19:51, Apr. 2011.

[2] J. A. Stankovic, "Research challenges for wireless sensor networks," SIGBED Rev., vol. 1, pp. 9-12, July 2004.

[3] B. Kitchenham and S. L. Pfleeger, "Principles of survey research: part 5: populations and samples," SIGSOFT Softw. Eng. Notes, vol. 27, pp. 17-20, September 2002.

[4] K. Doddapaneni, E. Ever, O. Gemikonakli, I. Malavolta, L. Mostarda, and H. Muccini, "A model-driven engineering framework for architecting and analysing Wireless Sensor Networks," in SESENA, 2012, pp. $1-7$.

[5] M. Kasunic, "Designing an Effective Survey," Handbook CMU/SEI-2005-HB-004, Software Engineering Institute, Carnegie Mellon University, Tech. Rep., 2005. [Online]. Available: www.sei.cmu.edu/pub/documents/05.reports/pdf/05hb004.pdf

[6] L. Kish, Survey sampling, ser. Wiley Classics Library. Wiley, 1995. [Online]. Available: http://books.google.it/books?id=yoppAAAAMAAJ
[7] Y. Ben Maissa, F. Kordon, S. Mouline, and Y. Thierry-Mieg, "Modeling and analyzing wireless sensor networks with verisensor: An integrated workflow," in Transactions on Petri Nets and Other Models of Concurrency VIII, ser. Lecture Notes in Computer Science, M. Koutny, W. Aalst, and A. Yakovlev, Eds. Springer Berlin Heidelberg, 2013, vol. 8100, pp. 24-47. [Online]. Available: http://dx.doi.org/10.1007/978-3-642-40465-8_2

[8] L. Samper, F. Maraninchi, L. Mounier, and L. Mandel, "Glonemo: Global and accurate formal models for the analysis of ad-hoc sensor networks," in Proceedings of the First International Conference on Integrated Internet Ad Hoc and Sensor Networks, ser. InterSense '06. New York, NY, USA: ACM, 2006. [Online]. Available: http://doi.acm.org/10.1145/1142680.1142684

[9] L. Samper, F. Maraninchi, and E. Jahier, "On the importance of modeling the environment when analyzing sensor networks," in Sensor and Ad Hoc Communications and Networks, 2006. SECON '06. 2006 3rd Annual IEEE Communications Society on, vol. 3, Sept 2006, pp. 835-841.

[10] M. Pan, C. Tsai, and Y. Tseng, "Emergency guiding and monitoring applications in indoor 3d environments by wireless sensor networks," Int. J. Sen. Netw., vol. 1, no. 1/2, pp. 2-10, Sep. 2006. [Online]. Available: http://dx.doi.org/10.1504/IJSNET.2006.010829

[11] A. Koubâa and M. B. Jamâa, "Taxonomy of fundamental concepts of localization in cyber-physical and sensor networks," Wirel. Pers. Commun., vol. 72, no. 1, pp. 461-507, Sep. 2013. [Online]. Available: http://dx.doi.org/10.1007/s11277-013-1025-z

[12] P. Barsocchi, S. Lenzi, S. Chessa, and G. Giunta, "Virtual calibration for rssi-based indoor localization with ieee 802.15.4," in Communications, 2009. ICC 'O9. IEEE International Conference on, June 2009, pp. 1-5.

[13] T. He, C. Huang, B. M. Blum, J. A. Stankovic, and T. Abdelzaher, "Range-free localization schemes for large scale sensor networks," in Proceedings of the 9th Annual International Conference on Mobile Computing and Networking, ser. MobiCom '03. New York, NY, USA: ACM, 2003, pp. 81-95. [Online]. Available: http://doi.acm.org/10.1145/938985.938995

[14] 2012, autoCAD website. [Online]. Available: http://usa.autodesk.com/ autocad/

[15] D. C. Schmidt, "Guest editor's introduction: Model-driven engineering," Computer, vol. 39, no. 2, pp. 25-31, Feb. 2006. [Online]. Available: http://dx.doi.org/10.1109/MC.2006.58 